\title{
Características Morfológicas de la Retina del Calderón Común (Globicephala melas; Traill, 1809) y su Relación con el Hábitat
}

\author{
Morphological Characteristics of Pilot Whales Retina (Globicephala \\ melas; Traill, 1809) and their Relationship to Habitat
}

Mengual, R.*; Segovia, Y.* \& García, M.*

MENGUAL, R.; SEGOVIA, Y. \& GARCÍA, M. Características morfológicas de la retina del calderón común (Globicephala melas; Traill, 1809) y su relación con el hábitat. Int. J. Morphol., 32(4):1399-1406, 2014.

RESUMEN: Existe una estrecha relación entre las características morfológicas de los sistemas sensoriales, su funcionamiento y el hábitat al que están adaptados los organismos. En este sentido, de todos los mamíferos marinos estudiados, los cetáceos son los que más profundamente han modificado su estructura y fisiología ocular por su estrecha adaptación a una vida exclusivamente acuática. Para aportar más datos a la literatura, el objetivo de este trabajo es describir morfológicamente la retina de la ballena piloto a través de técnicas de microscopia óptica, con el fin de relacionarla con su adaptación al medio acuático. Nuestros datos muestran que la retina de Globicephala melas se organiza de acuerdo al mismo plan básico de los vertebrados. Tiene un grosor medio alrededor de $330 \pm 23 \mu \mathrm{m}$ en las zonas de alta densidad de células ganglionares y $175 \pm 2 \mu \mathrm{m}$ en la zona periférica. La capa de los fotorreceptores se corresponde con el $45 \%$ del grosor de la retina total. Presenta largos segmentos externos. La capa más característica de cetáceos en general y de Globicephala melas en particular, es la capa de células ganglionares. Su grosor, de 77,76 37,26 siendo la más variable de toda la retina. Esta capa presenta baja densidad celular pero tamaños excepcionalmente grandes, de 10 a $75 \mu \mathrm{m}$ (promedio de 33,5 $\mu \mathrm{m}$ ), denominadas células ganglionares gigantes.

PALABRAS CLAVE: Globicephala melas; Visión de cetáceos; Retina.

\section{INTRODUCCIÓN}

En los vertebrados la visión es uno de los principales sentidos mediante el cual los organismos perciben e interpretan su entorno. El proceso visual extrae información a partir de la luz que reflejan los objetos hacia el ojo y suministra datos de vital importancia para la supervivencia del organismo. Sin embargo, el tipo de información extraída a partir del entorno depende de las necesidades específicas de cada organismo, de forma que podemos decir que cada organismo vive en un mundo sensorial particular como consecuencia de su adaptación al medio y a su modo de vida.

En los vertebrados, la visión se inicia cuando la luz que atraviesa la córnea y el cristalino alcanza la retina, una delgada y transparente estructura laminar de tejido neural situada en la parte posterior del globo ocular, cuya función es recoger los estímulos luminosos, elaborar y transmitir las sensaciones visuales. Debido al origen embrionario de la retina, los fotorreceptores, las células que contienen los pigmentos visuales y que, por tanto, inician la respuesta visual, contactan directamente con el epitelio pigmentario. En cambio, las células ganglionares, cuyos axones constituyen el nervio óptico, se hallan más cerca de la córnea. Como consecuencia, la luz debe atravesar las capas proximales de la retina antes de alcanzar los fotorreceptores situados en la parte distal. Estas células transforman los fotones en señales nerviosas y, a través de una elaborada red de sinapsis, la información es transmitida por el nervio óptico hasta el cerebro donde se interpreta. Este proceso permite que el sistema visual obtenga información sobre el movimiento, color $\mathrm{y}$ forma del mundo exterior.

Todas las retinas de vertebrados se organizan básicamente en dos capas sinápticas, la capa plexiforme externa o distal y la capa plexiforme interna o proximal, intercaladas entre tres capas celulares, externa, interna, y capa de células ganglionares. El pericarion de los fotorreceptores se localiza en la capa nuclear externa, mientras que en la capa nuclear interna se sitúan las células horizontales, las células bipolares y las células amacrinas e interplexiformes. El pericarion de las células ganglionares constituye la capa más 
proximal de la retina, la capa de las células ganglionares. Por su parte, la capa plexiforme externa contiene los procesos de los fotorreceptores, células horizontales, células bipolares e interplexiformes, mientras que en la capa plexiforme interna interaccionan procesos de células bipolares, amacrinas, interplexiformes y ganglionares.

Aunque la retina de los vertebrados muestra una estructura histológica muy conservada filogenéticamente, la extraordinaria diversidad de hábitats, comportamientos y modos de vida da lugar a que cada clase taxonómica presente una serie de particularidades como consecuencia de su adaptación al medio. Desde los peces abisales, pasando por los vertebrados nocturnos a las aves, para las que la visión es primordial, dichas características distintivas suelen limitarse a diferencias celulares y moleculares como consecuencia del rango espectral y de la intensidad de la luz disponible. Por ejemplo: 1) El espesor relativo de la capa de los fotorreceptores respecto a la retina interna es mayor en especies que habitan en lugares con elevada intensidad de luz. 2) La morfología, tipo y número de fotorreceptores varía según las especies dependiendo de su adaptación a diferentes ambientes fóticos. La mayor parte de vertebrados nocturnos o que viven en ambientes con intensidades bajas de luz poseen retinas ricas en bastones, mientras que aquellos que habitan en ambientes con abundante iluminación poseen un elevado porcentaje de conos. 3) El tipo de pigmentos visuales es una característica distintiva de las especies, dado que la discriminación del color depende de las características espectrales del ambiente en el que habitan. 4) La morfología de la retina interna, en cuanto al número de células de la capa nuclear interna muestra considerables diferencias entre las distintas especies. En aquellas adaptadas a ambientes escotópicos, el número de células bipolares y amacrinas es muy bajo si se compara con las que viven en condiciones de altos niveles de iluminación y con una visión en color desarrollada. 5) El número, distribución, subclases morfológicas de las células y sus respuestas fisiológicas, así como de sus conexiones sinápticas es diferente en distintos grupos taxonómicos.

Sin embargo, estudios comparativos de la retina de los vertebrados han llevado a la conclusión de que las diferencias en la estructura de la retina no se deben tanto a la clasificación taxonómica como a los requerimientos funcionales que los factores ecológicos y etológicos imponen al sistema visual (Wagner, 1990). De esta manera, especies no emparentadas que viven en la misma zona fótica pueden presentar notables similitudes en sus sistemas visuales. Por ejemplo, la retina los vertebrados nocturnos y los mamíferos marinos presentan retinas con características similares que las diferencian de la retina de los vertebrados diurnos. Esto apoya la idea de que la organización de la retina está íntimamente relacionada con el estilo de vida (Hughes, 1975).

En este sentido, los mamíferos marinos, que provienen de antepasados terrestes, presentan una oportunidad única para examinar el curso de la evolución de los procesos visuales en su adaptación desde el medio terrestre al acuático. Este medio, debido a sus propiedades lumínicas condiciona la visión de los mamíferos marinos. Estas propiedades incluyen el incremento de la densidad óptica del agua con respecto al aire, la dispersión y difracción de la luz por las partículas en suspensión, la marcada atenuación de la luz en el agua (pérdida de energía radiante), la diferencia de luminosidad a diferentes profundidades o en diferentes ángulos de visión y la alteración de la composición espectral con la profundidad a través de la absorción selectiva de la longitud de onda. A pesar de estos condicionantes, la visión debe de jugar un importante papel en la vida de los mamíferos marinos para la rápida localización de objetos a distancia y una exploración detallada de su entorno próximo: reconocimiento madre-hijo o a compañeros, seleccionar la comida y comunicarse por medio de pautas de comportamiento corporal y el lenguaje.

De todos los mamíferos marinos estudiados, los cetáceos son los que más profundamente han modificado su estructura y fisiología por su estrecha adaptación a una vida exclusivamente acuática. Constituyen, sin duda, un buen ejemplo de la versatilidad de la vida y de cómo, con estructuras diferentes, se pueden lograr adaptaciones muy perfeccionadas al mismo hábitat: siguen una línea de conservación del patrón mamífero pero modificado por la presión del medio ambiente (Dawson, 1988). Estas adaptaciones convergen con las de otros vertebrados como los peces que desarrollan su vida en el mismo hábitat. Sin embargo, y dado que son mamíferos originariamente terrestres, no se han desprendido totalmente de las características que permiten la visión en el aire (Dawson). Así, tanto los mecanismos ópticos como la retina de los mamíferos marinos, muestran adaptaciones específicas para la visión aérea y submarina (Dral, 1972).

La estructura de la retina de cetáceos se ha estudiado en varias especies, como en el delfín mular (Tursiops truncatus) (Perez et al., 1972; Dawson \& Perez, 1973; Dral, 1977; Dawson et al., 1982), el delfín común (Delphinus delphis) (Dral, 1983), la marsopa de Dall (Phocenoides dalli) (Murayama et al., 1992, 1995), la beluga (Delphinapterus leucas) (Pillery, 1964), el rorcual común (Balaenoptera physalus) (Pillery \& Wandeler, 1964), y el rorcual aliblanco (Balaenoptera acutorostrata) (Murayama et al., 1992; Mass \& Supin, 2007). Estas investigaciones han confirmado que la retina es cualitativamente similar a la de los mamíferos 
terrestres nocturnos, debido a la predominancia de bastones respecto de conos, con una proporción de conos de únicamente del 1\% (Peichl et al., 2001).

En este trabajo estudiamos las características estructurales de la retina de la ballena piloto, Globicephala melas, con el fin de aportar nuevos datos acerca de la visión de los cetáceos y relacionarla con su hábitat y modo de vida.

\section{MATERIAL Y MÉTODO}

Animal de estudio. Para la realización del trabajo hemos utilizado 2 ojos de 2 dos individuos diferentes de Globicephala melas (Traill, 1809), llamado comúnmente calderón común o de aleta larga y también ballena piloto.

Es un odontoceto de tamaño mediano, de forma alargada, esbelto y con un pedúnculo caudal grueso; los machos adultos tienen de 4,5 a 6 metros de longitud, aunque pueden alcanzar los $8,5 \mathrm{~m}$; las hembras miden unos 4-5 $\mathrm{m}$, aunque pueden llegar a medir $6 \mathrm{~m}$. El peso oscila de 1,8 a 3,5 toneladas en los individuos adultos. Las crías miden al nacer $1,5-1,9 \mathrm{~m}$, y pesan alrededor de $75 \mathrm{~kg}$.

El régimen alimentario varía según el área geográfica y la concentración estacional de las presas, por lo que efectúan migraciones periódicas. Las inmersiones de alimentación suelen durar unos 10 minutos; por lo general realiza varias inspiraciones rápidas y luego se sumerge. Generalmente los cefalópodos constituyen la mayor parte de su alimento, pero también consumen peces de diversas especies. Estas exigencias alimenticias les conducen a frecuentar aguas relativamente profundas, generalmente situadas más allá de la isobata de los 200 m, pudiendo llegar a bajar hasta 600 metros de profundidad.

El calderón común es una especie típicamente gregaria: los grupos están formados generalmente por 10-50 individuos, pero se pueden formar concentraciones de centenares de animales. El área de distribución de la especie cubre la mayor parte de los océanos templados fríos o subsolares. En Europa, la distribución se extiende desde el Mediterráneo hasta Groenlandia, Islandia y Noruega, con una zona de concentración al norte de las islas Británicas, en especial en las islas Faroe.

Obtención y procesamiento de los ojos de Globicephala melas. Las muestras se tomaron en las Islas Faroe, donde está permitida la caza controlada de odontocetos, especialmente de la ballena piloto. Después del sacrificio de los animales, en un tiempo no mayor a dos horas y una temperatura ambiental inferior a $10^{\circ} \mathrm{C}$, procedimos a la extracción de los globos oculares. Se eliminó la porción anterior del globo ocular, incluyendo córnea, iris, cristalino y la mayor parte de humor vítreo y se procedió a la fijación por inmersión de las copas ópticas, retina incluida, con formaldehido al 10\%, en tampón fosfato $0,1 \mathrm{M}$ a pH 7,4.

Para su estudio histológico, las retinas se dividieron en cuadrantes (temporal, nasal, dorsal y ventral) y de cada uno, la zona central, media, periférica y la zona de alta densidad en células ganglionares, se incluyeron en Epon-812. La estructura general de la retina y el grosor de cada una de las capas fueron analizadas a partir de cortes semifinos teñidos con azul de tolouidina. Finalmente, para la observación y captura de imágenes de los cortes transversales de la retina se utilizó el microscopio Leica DMRB, con el que obtuvimos planos generales de la retina completa y detalles de las diferentes capas.

\section{RESULTADOS}

\section{Estructura general de la retina de Globicephala melas.}

La retina de Globicephala melas, se organiza de acuerdo al mismo plan básico de los vertebrados (Fig. 1). Desde el epitelio pigmentario, sus células se disponen en tres capas donde se localizan los núcleos, y establecen sinapsis en dos capas sinápticas o plexiformes. El pericarion de los fotorreceptores se localiza en la capa nuclear externa, mientras que el de células horizontales, bipolares, amacrinas y células de Müller lo hace en la capa nuclear interna. El pericarion de las células ganglionares constituye la parte más proximal de la retina, la capa de las células ganglionares, cuyos axones constituyen la capa de fibras del nervio óptico. La capa plexiforme externa contiene los procesos de los fotorreceptores, células horizontales y células bipolares. En la capa plexiforme interna interaccionan procesos de células bipolares, amacrinas y ganglionares.

En la capa de fotorreceptores (Fig. 1), se puede apreciar un denso empaquetamiento celular. La capa nuclear externa presenta los núcleos de los fotorreceptores dispuestos en $8 \pm 2$ filas. La mayor parte de ellos presentan una cromatina densamente empaquetada, lo que nos lleva a suponer que corresponden a los núcleos de los bastones. Algunos núcleos próximos a la membrana limitante externa presentan aspecto vesicular, por lo que podrían corresponder a los núcleos de los conos, no obstante esto no se puede demostrar con las técnicas convencionales de microscopia óptica. Los largos segmentos externos miden aproximadamente $37,5 \pm 14,9 \mu \mathrm{m}$. 


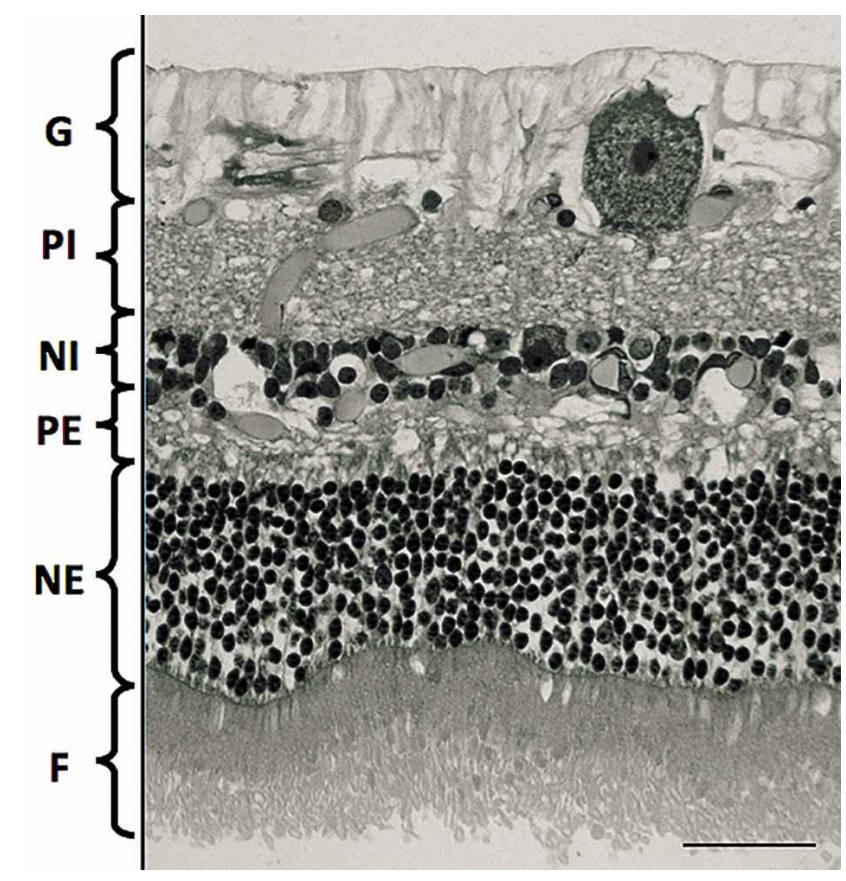

Fig. 1. Imagen de un corte transversal de retina de calderón común. G: capa de las células ganglionares; PI: capa plexiforme interna; NI: capa nuclear interna; PE: capa plexiforme externa; F: fotorreceptores. NE: capa nuclear externa; F.NO: capa de las fibras del nervio óptico. Barra=25 $\mu \mathrm{m}$.

La capa plexiforme externa constituye una estrecha lámina sináptica de de $14,39 \pm 1,47 \mu \mathrm{m}$. Un hecho típico de esta especie es la presencia de pequeños vasos en esta capa.

La capa nuclear interna (Fig. 2), de 34,3 $\pm 5,03 \mu \mathrm{m}$ de espesor, se halla también vascularizada. En su parte más distal, presenta el citoplasma de las células horizontales; en la parte central se localiza el soma de las células bipolares (Fig. 2, flechas negras) que se identifica con el microscopio óptico por constituir un anillo de citoplasma alrededor del núcleo, redondo y fuertemente teñido, aunque existen algunos tipos de estas células con grandes somas y de citoplasma abundante y, en el borde interno de la capa nuclear interna observamos células redondas con un abundante citoplasma y núcleo débilmente tenido; dada su localización, probablemente se corresponden con las células amacrinas (Fig. 2, flechas discontinuas).

La capa plexiforme interna, con un grosor de 36,8 $\pm 3,99$ $\mu \mathrm{m}$, es similar al de la capa nuclear interna (Fig. 3). Por último, la capa de las células ganglionares, cuyos somas se localizan en una sola hilera, es con diferencia, la capa más característica de los cetáceos, dada sus diferencias con el resto de los mamíferos (Fig. 3). Su grosor, de 77,76 $\pm 37,26 \mu \mathrm{m}$ es el más variable de toda la retina.

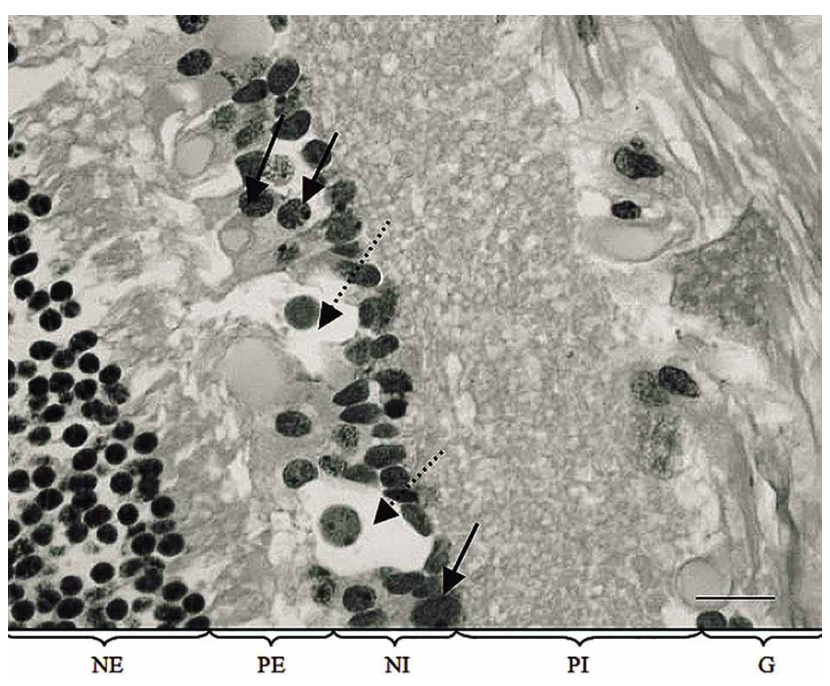

Fig. 2. Detalle de la capa plexiforme externa y la capa nuclear interna. G: capa de las células ganglionares y fibras del nervio óptico; PI: capa plexiforme interna; NI: capa nuclear interna; PE: capa plexiforme externa; NE: capa nuclear externa (fotorreceptores). Barra $=10 \mu \mathrm{m}$.

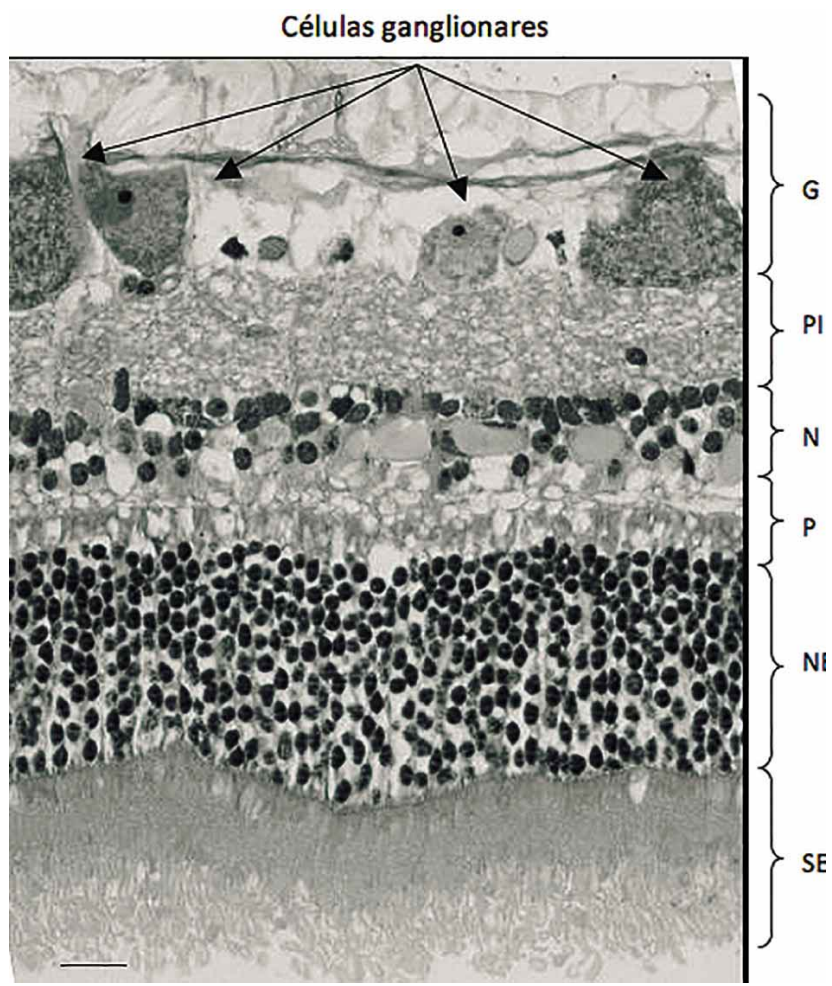

Fig. 3. Micrografía transversal de la retina donde se aprecian las células ganglionares dispuestas en una hilera. G: capa de las células ganglionares y fibras del nervio óptico; PI: capa plexiforme interna; NI: capa nuclear interna; PE: capa plexiforme externa; NE: capa nuclear externa; SE: segmentos externos de los fotorreceptores. Barra $=10 \mu \mathrm{m}$. 
Otras características de la capa de las células ganglionares, es que dichas células presentan una baja densidad pero tamaños excepcionalmente grandes de 10 a 75 $\mu \mathrm{m}$ (promedio de $33,5 \mu \mathrm{m}$ ), lo que lleva a denominarlas células ganglionares gigantes.

En la Figura 4, podemos observar el grosor de la retina y cada una de sus capas, medido en la zona central, media, de alta densidad y periférica.

El grosor de la retina presenta una gran variación entre las diversas regiones: de aproximadamente $175 \pm 2 \mu \mathrm{m}$ en la zona periférica y $330 \pm 23 \mu \mathrm{m}$ en la zona de alta densidad de células ganglionares. El espesor relativo de la capa de los fotorreceptores respecto al grosor medio total de la retina, es de $45 \%$ aproximadamente.

Nuestros resultados acerca de las diferencias en grosor de la retina según diferentes zonas, indican que:

- La retina periférica muestra una considerable disminución respecto a las zonas central, media y de alta densidad de células ganglionares. Dicha disminución se debe, sobre todo a que la capa de células ganglionares y, de los fotorreceptores y la capa nuclear externa presentan una marcada reducción. Inversamente, la capa de fibras del nervio óptico va aumentando en grosor a medida que nos acercamos a dicho nervio.

- Las capas nuclear interna y plexiforme interna, no presentan variaciones significativas en grosor a excepción de la zona periférica donde son ligeramente más delgadas.

- La capa plexiforme externa es prácticamente invariable en grosor, aunque el mínimo grosor se presenta en la parte periférica.

\section{DISCUSIÓN}

Nuestros datos muestran que la retina de Globicephala melas tiene un grosor medio (alrededor de $330 \pm 23 \mu \mathrm{m}$ ) inferior al de la retina de otros cetáceos, cuyo grosor de puede alcanzar las 425 micras (Dral, 1977; Dawson et al.; Murayama et al., 1995). Los datos publicados acerca del grosor de la retina de mamíferos indican que existe una gran variación, desde menos de $100 \mu \mathrm{m}$ en los mamíferos con retinas avasculares, como algunos marsupiales, a más de $300 \mu \mathrm{m}$ en los que poseen retinas vasculares (Buttery et al. 1991). El motivo del mayor grosor de la retina de cetáceos podría ser el hecho de que es vascular, dado que, en general, las retinas vasculares presentan un mayor grosor respecto a aquellas que no lo son (Buttery et al.). Aunque se ha especulado mucho acerca del papel de la vascularización de la retina, en el caso de los cetáceos, ésta podría ejercer un importante papel en el mantenimiento de la temperatura de este tejido nervioso (Dawson et al.).

En cuanto al espesor relativo de la capa de los fotorreceptores respecto al grosor medio total de la retina, es de $45 \%$ aproximadamente. Este valor es similar al de otros mamíferos: $40-42 \%$ en especies vasculares y el $44-47 \%$ en especies avasculares (Buttery et al.). En esta capa es de destacar la abundancia de bastones, un rasgo común entre los animales que habitan en lugares con escasa iluminación, con la finalidad de incrementar la sensibilidad (Levenson \& Schusterman, 1999). Peichl et al. han publicado que Globicephala melas tiene, aproximadamente, 330.000 bastones $/ \mathrm{mm}^{2}$. También, probablemente, con la finalidad de incrementar la sensibilidad, la retina de Globicephala melas presenta largos segmentos externos, de aproximadamente $37,5 \pm 14,9 \mu \mathrm{m}$, que incrementarían el área disponible para

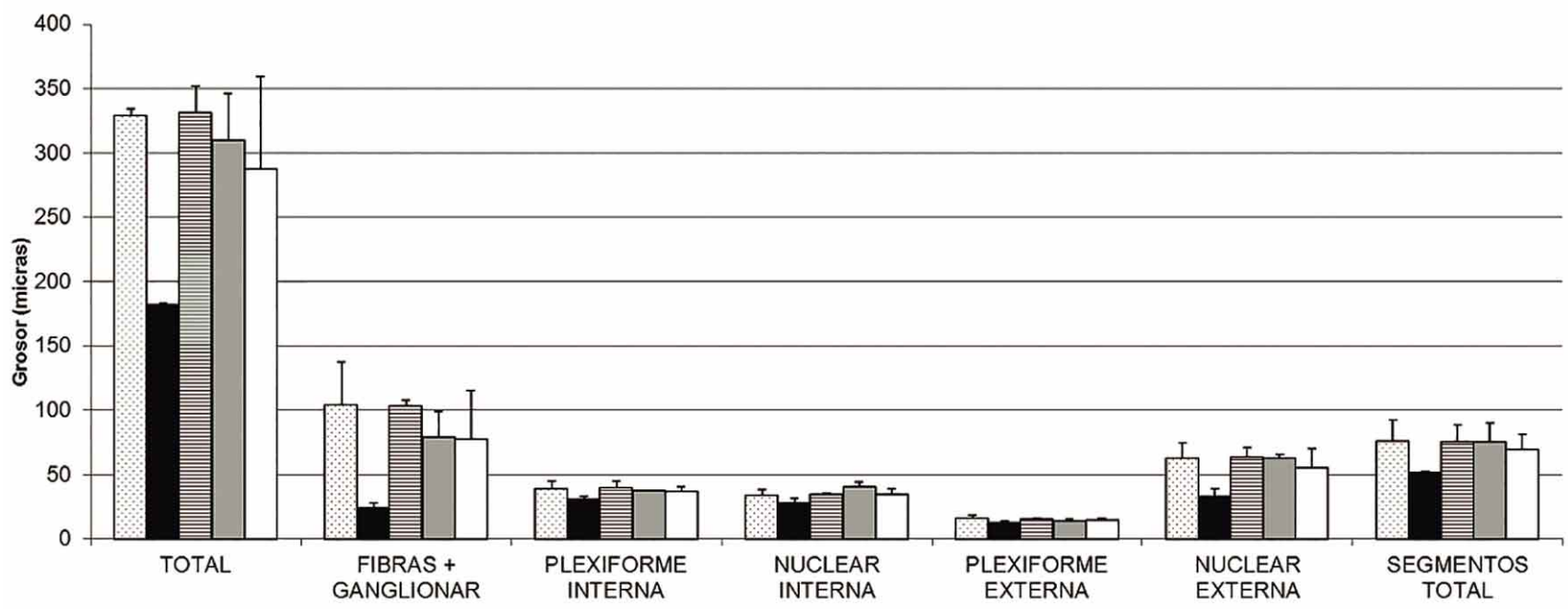

Fig. 4. Grosor de las distintas capas de la retina en distintas zonas de la misma. 
la localización de los pigmentos visuales, lo cual, a su vez, aumentaría la absorción de la luz.

Por otra parte, el número de conos es mucho menor que el de bastones. Esto ha sido comprobado por Peichl et al., en un estudio realizado en varias especies de mamíferos marinos, donde los conos, en general, representan un 1,5\% del total de fotorreceptores. Esta proporción es comparable a la hallada en otros cetáceos y en algunos mamíferos terrestres crepusculares o con fases de actividad diurna y nocturna, tales como la rata, el ratón, el conejo y el gato (Peichl et al.).

La baja presencia de conos es una característica que comparten la mayor parte de las especies de cetáceos (Dawson). Además, Peichl et al. han demostrado, mediante inmunorreactividad frente a las opsinas específicas de los conos L (sensibles a longitudes de onda medias o largas) y de los conos $\mathrm{S}$ (sensibles a longitudes de onda cortas), que muchos mamíferos marinos, entre ellos varias ballenas y focas, no poseen conos $\mathrm{S} y$, por tanto, son monocromáticos. No se conoce el significado funcional de este hecho, ya que la mayoría de los mamíferos terrestres, aun siendo nocturnos, presentan visión en color. Además, los cetáceos han perdido los conos sensibles a las longitudes de onda corta, que es justamente la que penetra a más profundidad y hacia la que se desplazan algunos conos en los peces teleósteos. Aun no siendo clave para los cetáceos la visión en color, la pérdida de los conos S podría suponer una desventaja para la discriminación del brillo (Muntz, 1975) y el contraste (Lythgoe, 1975) cuando los objetos son más oscuros que el fondo, cuando son brillantes u oscuros en la profundidad, a grandes distancias, o son vistos desde abajo.

La capa plexiforme externa, de 14,39 $\pm 1,47$ micras, es la capa más delgada de la retina de Globicephala melas y en todas las especies estudiadas. Además, es la capa que menos variaciones de grosor presenta en todas las especies de vertebrados, tanto en aquellas que presentan retinas vasculares como las de retinas avasculares (Buttery et al.). Ya que la resolución espacial se determina principalmente en esta capa, la agudeza visual no estaría influenciada por el grosor de la retina (Buttery et al.).

En cuanto a la morfología de la retina neural, especialmente de la capa nuclear interna, plexiforme interna y capa de células ganglionares, muestra considerables diferencias entre las distintas especies de vertebrados y la interpretación funcional de estas características es más difícil que en el caso de losfotorreceptores. La retina interna de Globicephala melas es un 60\% del total debido a la superficie que ocupa la capa de células ganglionares, no al grosor de las capas nuclear interna y plexiforme interna, responsa- bles del procesamiento de la información visual que es conducida hacia los centros superiores.

La capa nuclear interna, de 34,3 $\pm 5,03 \mu \mathrm{m}$ de espesor es compatible con la de las especies de mamíferos que presentan vascularización intrarretiniana (Buttery et al.). Como consecuencia del espesor de la capa nuclear interna, el de la capa plexiforme interna es algo mayor $36,8 \pm 3,99 \mu \mathrm{m}$, como ocurre en todas las retinas de vertebrados descritas. Sin embargo, si la comparamos con el grosor de la capa plexiforme interna de otras especies de mamíferos (Buttery et al.) como la rata $(59,9 \pm 1,3)$, el grosor es menor. Si consideramos que esta capa tiene funciones relacionadas con la resolución temporal y la detección del movimiento (Dowling, 1987), podríamos deducir, a partir de estos datos, que estas capacidades son menores que las de mamíferos terrestres. Cuando la comparamos con la retina de teleósteos $(45,0 \mu \mathrm{m}$, García \& De Juan, 1999) el valor de esta capa es muy similar.

En cuanto a la capa de células ganglionares junto con la capa de fibras del nervio óptico, es de 77,76 $\pm 37,26$, un grosor mucho mayor que el de hallado en otros mamíferos no cetáceos debido a la presencia de las células ganglionares gigantes. Dichas células han sido descritas en todos los mamíferos marinos estudiados a excepción del delfín de río Platanista gangética, cuyas células miden alrededor de 20 micras: Balaenoptera physalus (Pillery \& Wandeler, 1964), Tursiops truncatus (Perez et al.; Dawson et al.; Mass \& Supin, 1995), Delphinus delphis (Dral, 1983), Phocoena phocoena (Mass et al., 1986), Balaenoptera acutorostrata (Murayama et al., 1992), Eschrichtius gibosus (Mass \& Supin, 1997). El tamaño de estas células contrasta con el de los mamíferos terrestres, de aproximadamente $25 \mu \mathrm{m}$ en algunos casos (Hughes, 1975, 1981). En cambio, en otros vertebrados acuáticos como los peces, no se ha observado un gran tamaño de las células ganglionares. Aunque todavía no tenemos explicación para la presencia de estas células, las células ganglionares gigantes en los mamíferos marinos se habrían originado para incrementar la sensibilidad de la retina en condiciones de luz escotópicas. Efectivamente, el gran tamaño de las células, con grandes campos dendríticos, incrementaría el número de fotorreceptores que convergen en una célula ganglionar; sin embargo disminuiría el poder de resolución. Por otra parte, estas células gigantes han sido relacionadas con las llamadas células ganglionares alfa, que según los estudios electrofisiológicos responden al movimiento y conducen los estímulos rápidamente de modo que permitirían a los cetáceos la detección rápida de presas o depredadores en un ambiente en que tanto la intensidad de la luz como el rango espectral no permiten captar otro tipo de estímulos. 
Respecto al grosor de la retina en función de la región, la zona de máxima densidad de células ganglionares es la más gruesa. Esto puede ser explicado por el hecho de que una elevada densidad de células ganglionares se halla relacionada con un denso empaquetamiento de los fotorreceptores, así como de células bipolares y otras interneuronas asociadas. Lo contrario podemos decir de la zona periférica: la disminución de fotorreceptores conlleva la consiguiente disminución de células ganglionares asociados a ellos y de interneuronas. De manera similar, la capa plexiforme interna muestra una reducción significativa en la parte periférica debido a que la disminución de fotorreceptores y células ganglionares implica una disminución de interneuronas y de contactos sinápticos. Por último, a medida que los axones de las células ganglionares se dirigen al nervio óptico, la zona central de la retina va incrementando el grosor de dicha capa.
En conclusión, la retina de Globicephala melas presenta una retina típica de mamíferos con las siguientes características que le permiten la visión en el ambiente acuático con una baja iluminación:

I. La capa de los fotorreceptores, casi exclusivamente formada por bastones, permitiría la captación de bajas intensidades de luz como adaptación a ambientes escotópicos. Asimismo estos fotorreceptores presentan largos segmentos externos, hecho relacionado con el aumento de absorción de luz.

II. La presencia de células ganglionares gigantes con amplios campos dendríticos para aumentar la sensibilidad a la luz, aunque con la consiguiente pérdida de poder de resolución.

MENGUAL, R.; SEGOVIA, Y. \& GARCÍA, M. Morphological characteristics of pilot whales retina (Globicephala melas; Traill, 1809) and their relationship to habitat. Int. J. Morphol., 32(4):1399-1406, 2014.

SUMMARY: There is a close relationship between morphological features of sensory systems, their function and habitat to which these organisms are adapting. In this sense, of all marine mammals that have been studied, cetaceans are the ones that have profoundly changed structure and ocular physiology in their adaptation to an exclusively aquatic life. To add further data to the literature, the aim of this paper is to describe morphologically the retina of the pilot whale through optical microscopy and relate their adaptation to the aquatic environment. Our data show that the retina of the long-finned pilot whale is organized according to the same basic plan of vertebrates. It has an average thickness of about $330 \pm 23$ microns in areas of high ganglion cell density of $175 \pm 2$ microns in the peripheral zone. Photoreceptor layer corresponds to $45 \%$ of total thickness of the retina and has long outer segments. The most significant characteristic of cetaceans in general and long-finned pilot whale in particular, is the ganglion cell layer. Thickness of $77.76 \pm 37.26$ being the most variable of the entire retina. This layer has a low density but exceptionally large cell size of 10 to 75 microns (average of 33.5 microns), known as giant ganglion cells.

KEY WORDS: Globicephala melas; Cetacean vision; Retina.

\section{REFERENCIAS BIBLIOGRÁFICAS}

Buttery, R. G.; Hinrichsen, C. F.; Weller, W. L. \& Haight, J. R. How thick should a retina be? A comparative study of mammalian species with and without intraretinal vasculature. Vision Res., 31(2):169-87, 1991.

Dawson, W. W. The cetacean eye. In: Herman, L. M. (Ed.). Cetacean behaviour: mechanisms and functions. New York, Willey, 1988. pp.53-100.

Dawson, W. W.; Hawthorne, M. N.; Jenkins, R. L. \& Goldston, R. T. Giant neural systems in the inner retina and optic nerve of small whales. J. Comp. Neurol., 205(1):1-7, 1982.

Dawson, W. W. \& Perez, J. M. Unusual retinal cells in the dolphin eye. Science, 181(4101):747-9, 1973.

Dowling, J. E. The retina: an approachable part of the brain. Cambridge, Harvard University Press, 1987. pp.275.
Dral, A. D. G. Aquatic and aerial vision in the bottle-nosed dolphin. Neth. J. Sea Res., 5(4):510-3, 1972.

Dral, A. D. G. On the retinal anatomy of Cetacea (mainly Tursiops truncatus). In: Harrison, R. J. (Ed.). Functional Anatomy of Marine Mammals. London, Academic Press, 1977. pp.81-134.

Dral, A. D. G. The retinal ganglion cells of Delphinus delphis and their distribution. Aquat. Mamm., 10(2):57-68, 1983.

García, M. \& de Juan, J. Fine structure of the retina of black bass, Micropterus salmoides (Centrarchidae, Teleostei). Histol. Histopathol., 14(4):1053-65, 1999.

Hughes, A. Population magnitudes and distribution of the major modal classes of cat retinal ganglion cell as estimated from HRP filling and a systematic survey of the soma diameter spectra for classical neurones. J. Comp. Neurol., 197(2):303-39, 1981. 
Hughes, A. A quantitative analysis of the cat retinal ganglion cell topograpy. J. Comp. Neurol., 163(1):107-28, 1975.

Levenson, D. H. \& Schusterman, R. J. Dark adaptation and visual sensitivity in shallow and deep-diving pinnipeds. Mar. Mamm. Sci., 15(4):1303-13, 1999.

Lythgoe, J. N. The structure and function of iridescent corneas in teleost fishes. Proc. R. Soc. Lond. B Biol. Sci., 188(1093):437$57,1975$.

Mass, A. M.; Supin, A. Ya. \& Severtsov, A. N. Topographic distribution of sizes and density of ganglion cells in the retina of a porpoise Phocoena phocoena. Aquat. Mamm., 12:95-102, 1986.

Mass, A. M. \& Supin, A. Ya. Ganglion cells topography of the retina in the bottlenosed dolphin, Tursiops truncatus. Brain Behav. Evol., 45(5):257-65, 1995.

Mass, A. M. \& Supin, A. Ya. Ocular anatomy, retinal ganglion cell distribution, and visual resolution in the gray whale, Eschrichtius gibbosus. Aquat. Mamm., 23(1):17-28, 1997.

Mass, A. M. \& Supin, A. Ya. Adaptive features of aquatic mammals' eye. Anat. Rec. (Hoboken), 290(6):701-15, 2007.

Murayama, T.; Fujise, Y.; Aoki, I. \& Ishii, T. Histological Characteristics and Distribution of Ganglion Cells in the Retinae of the Dall's Porpoise and Minke Whale. In: Thomas, J. A.; Kastelein, R. A. \& Supin, A. Ya. (Eds.). Marine Mammal Sensory Systems. New York, Springer US, 1992. pp.137-45.

Murayama, T.; Somiya, H.; Aoki, I. \& Ishii, T. Retinal ganglion cell size and distribution predict visual capabilities of Dall's porpoise. Mar. Mamm. Sci., 11(2):136-49, 1995.

Muntz, W. R. A. Behavioral studies of vision in a fish and possible relationships to the environment. In: Ali, M. A. (Ed.). Vision in fish. New Approaches in Research. New York, Springer US, 1975.

Peichl, L.; Behrmann, G. \& Kröger, R. H. For whales and seals the ocean is not blue: a visual pigment loss in marine mammals. Eur. J. Neurosci., 13(8):1520-8, 2001.

Perez, J. M.; Dawson, W. W. \& Landau, D. In air visual acuity of the bottlenose dolphin (Tursiops truncatus). Cetology, 11:111, 1972.

Pillery, G. Zur Morphologie des Auges von Weisswal Delphinapterus leucas (Pallas). Hvalrådets Skrifter, 47:1-16, 1964.

Pilleri, G. \& Wandeler, A. Ontogenese und functionelle Morphologie der Auges des Finnwals Balaenoptera physalus L. (Cetacea, Mysticeti Balaenopteridae). Acta Anat., 57(Suppl. 50):1-74, 1964.
Wagner, H. J. Retinal structure of fishes. In: Douglas, R. \& Djamgoz, M. (Eds.). The visual system of fish. New York, Springer Verlag, 1990. pp.109-58.

Dirección para Correspondencia:

Yolanda Segovia Huertas

Departamento de Biotecnología

Facultad de Ciencias

Universidad de Alicante

ESPAÑA

Email: yolanda.segovia@ua.es

Recibido: 22-07-2014

Aceptado: 22-09-2014 